\title{
Argumentative Patterns for Justifying Scientific Explanations
}

\author{
Jean H. M. Wagemans ${ }^{1}$
}

(C) The Author(s) 2015. This article is published with open access at Springerlink.com

\begin{abstract}
The practice of justifying scientific explanations generates argumentative patterns in which several types of arguments may play a role. This paper is aimed at identifying these patterns on the basis of an exploration of the institutional conventions regarding the nature, the shape and the quality of scientific explanations as reflected in the writings of influential philosophers of science. First, a basic pattern for justifying scientific explanations is described. Then, two types of extensions of this pattern are presented. These extensions are derived from philosophical accounts of requirements for the quality of explanations and the choice of the best explanation from a number of candidate explanations respectively. The description of the second extension will make clear how pragmatic argumentation plays a role in argumentative patterns within the scientific domain.
\end{abstract}

Keywords Abduction - Academic communication - Argumentative pattern . Causal argumentation - Inference to the best explanation · Pragma-dialectics . Pragmatic argumentation · Scientific explanation

\section{Introduction}

The nature of scientific explanations has been the subject of extensive research. ${ }^{1}$ Scholars from fields as diverse as philosophy, psychology, and sociology have described the form of reasoning that is involved in finding a scientific explanation,

\footnotetext{
${ }^{1}$ I would like to thank Frans van Eemeren and Francisca Snoeck Henkemans for their helpful comments on an earlier version of this paper. The present paper is an adapted and extended version of the one I presented at the ECA 2015 Conference on Argumentation held in Lisbon (Wagemans to appear).
}

Jean H. M. Wagemans

j.h.m.wagemans@uva.nl

1 Department of Speech Communication, Argumentation Theory, and Rhetoric, University of Amsterdam, Amsterdam, The Netherlands 
the cognitive circumstances under which such explanations are formed, and the social aspects that play a role in scientific practices respectively. ${ }^{2}$

Within the field of argumentation theory, the interest in academic discourse in general and scientific explanations in particular is relatively new. In comparison to the research that is carried out in the fields mentioned above, argumentation theoretical research concerning scientific explanations does not focus on the genesis of such explanations but on their justification, i.e. the way in which scientists support their claims with arguments. In this paper, I aim to describe the prototypical argumentative patterns arising when scientists provide such a justification for their explanation of an observed fact. ${ }^{3}$

Scientific communication within a specific field of expertise can be described as an expert-to-expert interaction that is aimed at convincing other scientists working in the same field of the acceptability of a claim that is new in the sense that it deviates in some way or another from the claims that are already accepted and together constitute the body of knowledge of that field. Scientific claims are predominantly descriptive in nature and contain a new explanation of observed facts (events, phenomena). In anticipation or reaction to doubt or criticisms raised by their peers, scientists may support their claims by referring to accepted methodological starting points, information concerning the content of the observations, as well as other information that is part of the existing body of knowledge of their field of expertise. In Sect. 2 of this paper, I explore the institutional conventions that shape this way of supporting scientific claims and provide a basic argumentative pattern for justifying scientific explanations.

Philosophers of science have not only studied the basic shape of scientific explanations, but have also contemplated the requirements for 'good' explanations and-in the case a selection from several candidate explanations is involved-the criteria for deciding what is the 'best' explanation. In Sect. 3, I will use their accounts of these requirements and criteria in order to describe two extensions of the basic argumentative pattern for justifying scientific explanations. The first extension is related to institutional conventions regarding the requirements for good explanations and the second one is related to institutional conventions regarding the criteria for selecting the best explanation from a number of candidate explanations.

In Sect. 4, I will discuss my findings and indicate the role of pragmatic argumentation in the argumentative patterns that can be identified within the scientific domain.

\section{Justifying Scientific Explanations}

In the field of the philosophy of science, the nature of scientific claims and theories is a much-debated issue. Curd and Cover (1998, pp. 767-804) provide an overview of this debate, which centers on Hempel's two models of scientific explanation. In

\footnotetext{
${ }^{2}$ For an overview of approaches and research regarding the subject see for example Hacket et al. (2008).

3 See van Eemeren (to appear) for a general explanation of the pragma-dialectical starting points regarding the research into argumentative patterns in various communicative domains.
} 
this section, I summarize their account of these models, on the basis of which I then identify a basic argumentative pattern for justifying scientific explanations.

Scientific claims have only relatively recently been conceptualized as explanations. While in the second half of the nineteenth century the influential philosopherscientist Duhem stated that 'a physical theory is not an explanation' but rather a 'system of mathematical propositions [...] which aim to represent [...] a set of experimental laws' (1998, p. 767, note 3), it is nowadays a commonplace to state that the central aim of scientific practice is to provide explanations for observed facts. One of the key figures in this transition of thinking about the nature of scientific claims and theories is the philosopher of science Hempel, who in the twentieth century described two different models of scientific explanation.

The first of these two models is put forward in an article written by Hempel in 1948 and is called the deductive-nomological (D-N) model of scientific explanation. This model assumes that the reasoning behind the explanation is deductively valid and therefore that the event to be explained - the explanandum-could have been predicted with certainty. There are however also explanations that are based on nondeductive reasoning and therefore do not enable the scientist to predict the explanandum with certainty but only with a specific degree of probability. Since such explanations, according to Hempel, also deserve to be labeled as scientific explanations, from 1962 onward he developed a second model of scientific explanation, which he called the inductive-statistical (I-S) model.

A characteristic of scientific explanations that is reflected in both of Hempel's models is that such an explanation contains an empirical law. This characteristic is expressed in the so-called 'covering law thesis' and it can, according to several influential philosophers of science, be taken a basic requirement for genuinely calling an explanation of an observed fact a 'scientific' explanation:

According to the covering law thesis, explanations are arguments (either deductively valid or inductively strong) that have among their premises at least one statement of an empirical law. [...] Hempel's covering law thesis [...] takes explanation to be essentially an inference showing that the explanandum event was to be expected. Given the information in the premises of the explanatory argument, the explanandum event could have been predicted, either with certainty (D-N) or with high probability (I-S). This capability of being used as a prediction is seen by many philosophers of science as the hallmark of a good explanation. For Hempel, Carnap, Nagel, and others, being able, at least in principle, to predict the event to be explained guarantees that the explanation has testable, empirical content and gets to the heart of what distinguishes genuine explanation from mere pseudoexplanation. (Curd and Cover 1998, p. 799)

I now turn to presenting the two models in more detail and translating them in argumentation theoretical terms. On the basis of this translation, I then identify a basic argumentative pattern that is generated when scientists provide scientific explanations of observed facts. 
Hempel's deductive-nomological (D-N) model is premised on the idea that apart from the requirement that a scientific explanation should contain an empirical law, several other requirements are at place:

The requirement that the explanans include at least one empirical law is obviously not sufficient for an explanation for two reasons. First, just including a law, any law, is not enough; the law must be essential to the derivation of the explanandum. Clearly, we would not be much impressed by a purported explanation in which the laws mentioned where entirely irrelevant to the event or phenomenon needing explanation. Second, all by themselves laws do not entail that any specific thing will happen. Thus, when we seek to explain the occurrence of an event, we must also include in the explanans statements of various initial conditions that, in conjunction with the laws, logically imply the explanandum. (Curd and Cover 1998, pp. 769)

The model has the explanandum (description of the event, law, or fact to be explained) as the conclusion and several elements that together constitute the explanans (statements of particular facts, initial conditions, and general laws) as premises. It covers those scientific explanations in which the conclusion follows from the premises with logical necessity and is schematized in the following way (Fig. 1).

As indicated above, a restriction of Hempel's deductive-nomological model of scientific explanation is that it only provides an account of those scientific explanations in which the conclusion follows from the premises with logical necessity. Since there are also scientific explanations in which the conclusion follows from the premises with a certain degree of probability rather than with logical necessity, Hempel later developed the inductive-statistical (I-S) model of scientific explanation. This model covers those scientific explanations that contain probabilistic laws and is schematized in the following way (Fig. 2).

Like the D-N model, the I-S model has the explanandum in the conclusion, stating that $a$ is $G(G a)$. It also has the premises together constituting the explanans, which in this case consists of a 'statistical law' expressing the probability $P$, ranging from zero to one $(0 \leq x \leq 1)$, of something being an $F$ also being a $G(G / F)$ and a 'particular fact' expressing that $a$ is $F(F a)$.

In order to enable a comparison with the argumentative patterns distinguished in the communicative domains that are studied in the other contributions to this special issue, I will now turn to translating Hempel's two models of scientific explanations into pragma-dialectical terms.

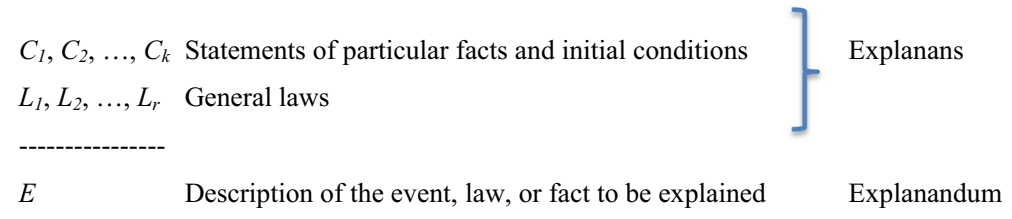

Fig. 1 General scheme of Hempel's D-N model of explanation (Curd and Cover 1998, p. 769) 


$\begin{array}{ll}P(G / F)=x & \text { Statistical law } \\ F a & \text { Particular fact } \\ \text {-------- } x & \end{array}$

Ga

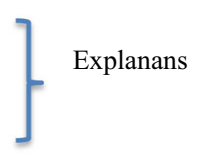

Explanandum

Fig. 2 General scheme of Hempel's I-S model of explanation (adapted from Curd and Cover 1998, p. 780)

Both models mention the particular fact (or facts) put forward in the expert-toexpert communication as part of the explanans. In the deductive-nomological model, this part is represented as $C_{1}, C_{2}, \ldots, C_{k}$ and in the inductive-statistical model as $F a$. Given that within the pragma-dialectical theory of argumentation, standpoints can be represented formal-linguistically as attributions of a specific predicate to a specific subject, I propose to translate this part of the models in the form of ' $\mathrm{X}$ is $\mathrm{Y}$ ' with ' $\mathrm{X}$ ' denoting the subject and ' $\mathrm{Y}$ ' denoting the predicate. A pragmatic representation of the standpoint in the basic pattern for justifying scientific explanations would then be something like: 'We may assume that a particular fact ( $\mathrm{X}$ is $\mathrm{Y}$ ) is the case'.

As to the explanandum, which has the argumentative function of justifying the explanans, a similar way of translating applies. While in the deductive-nomological model the explanandum is represented as $E$, in the inductive-statistical model it is represented more specifically as $G a$. Since the latter representation corresponds to the pragma-dialectical one, I propose to translate this part of the models as the main argument, the pragmatic representation of which would then be something like: 'It is observed that a (set of) particular fact(s) ( $\mathrm{X}$ is $\mathrm{Z}$ ) is the case'. 4

The elements of Hempel's models reflecting the 'covering law thesis' mentioned earlier in this section can be translated as an argument supporting what in pragmadialectics is called the 'justificatory force' of the argument. As part of the explanans, both types of laws establish a connection between the attribution of predicate ' $\mathrm{Z}$ ' to subject ' $\mathrm{X}$ ' and the attribution of predicate ' $\mathrm{Y}$ ' to subject ' $\mathrm{X}$ ' and therefore express the way in which the predicate of the argument is related to the predicate of the standpoint. The nature of this relation may differ with the field of research and the characteristics of the phenomena under scrutiny, which is reflected in the fact that Hempel felt the urge to distinguish between a deductive-nomological model, in which the relation between the particular facts in the explanans and the fact to be explained is deterministic, and a inductive-statistical model, in which this relation is probabilistic. Since the laws fulfill the same argumentative function in both models, this difference is not visible in the argumentative pattern as such. But this does not prevent the reconstructions of justifications of scientific explanations that are made with the help of the pattern from containing the relevant type of nomological relation.

\footnotetext{
4 Apart from being an adequate pragmatic representation of the explanandum in Hempel's models, this translation makes visible that Hempel's models of scientific explanation converge with Peirce's idea of abduction as reasoning from an observed fact to its explanation (see Wagemans 2014).
} 
Another difference between the two models is that the deductive-nomological model mentions 'initial conditions' as part of the explanans, while the inductivestatistical model only mentions a law. As explained earlier in this section, the reason for incorporating these initial conditions in the deductive-nomological model is that 'all by themselves laws do not entail that any specific thing will happen' (Curd and Cover 1998, pp. 769). Since there is no reason to assume that this additional condition does not hold for explanations in which probabilistic laws occur, I propose to ignore this difference in the pragma-dialectical translation of the models. In both cases, initial conditions can be conceived as arguments in support of the justificatory force of the main argument.

These considerations lead to the following proposal for a basic argumentative pattern for justifying scientific explanations (Table 1).

In this proposal of the basic pattern, the standpoint (1) represents part of the explanans of the two models $\left(C_{1}, C_{2}, \ldots, C_{k}\right.$ in as far as facts are expressed and $F a$ respectively) and the propositional content of the main argument (1.1) represents the explanandum ( $E$ and $G a$ respectively). The formal expression of the justificatory force of the argument $\left(1.1^{\prime}\right)$ is supported by two coordinative arguments $\left(1.1^{\prime} .1 \mathrm{a}\right.$ and $\left.1.1^{\prime} .1 \mathrm{~b}\right)$. The first of these arguments represents the law(s) $\left(L_{1}, L_{2}, \ldots, L_{r}\right.$ and $P(G /$ $F)=x$ respectively) and the second one the initial conditions $\left(C_{1}, C_{2}, \ldots, C_{k}\right.$ in as far as initial conditions are expressed). By translating these two elements of Hempel's models in this way, their argumentative function is conceived as supporting the relation between the main argument and the standpoint.

Given that the logical term 'conclusion' in most cases translates as 'standpoint' in the pragma-dialectical approach to argumentation, it may at first seem odd that the explanandum, which in Hempel's model appears as the conclusion, functions as the main argument. In scientific communication, however, it is not the explanandum but the explanans that is initially doubted or criticized and therefore in need for further support. The translation of the conclusion in Hempel's models as the main argument in the proposed basic argumentative pattern is therefore in line with the pragma-dialectical starting points regarding the felicity conditions of the speech acts of putting forward standpoints and arguments.

Table 1 Basic argumentative pattern for justifying scientific explanations

1 We may assume that a particular fact ( $\mathrm{X}$ is $\mathrm{Y})$ is the case

1.1 It is observed that a (set of) particular fact(s) (X is $\mathrm{Z}$ ) is the case

1.1' If it is observed that a (set of) particular fact(s) is the case, then we may assume that a particular fact is the case

1.1'.1a (Set of) law(s) S applies

1.1 '.1b (Set of) initial condition(s) T applies 


\section{Two Types of Extensions of the Basic Pattern}

Arguments, just like standpoints, may also be doubted and criticized, in which case they function as sub-standpoints. In addition to the arguments already mentioned in the basic pattern developed in the previous section, scientists may also put forward arguments in support of the explanandum, the laws, and the initial conditions. In this section, I discuss two types of such extensions of the basic pattern. Just as with the basic pattern, the extensions are derived from institutional conventions regarding the nature of scientific explanations as they are reflected in influential writings from the field of the philosophy of science.

The first type of extension is related to institutional conventions regarding the quality of the explanation. In identifying this extension, I make use of the additional requirements put forward by several philosophers of science in reaction to Hempel's idea that explanations should contain a law in order to genuinely be called scientific. The second type of extension is related to institutional conventions regarding criteria for choosing the best explanation from a number of candidate explanations. The description of this extension is based on an analysis of the role of abductive reasoning in scientific practices.

One of the main objections that were raised against Hempel's covering law models is known in the literature as the 'irrelevance objection' (Curd and Cover 1998, pp. 784-785; 800). This objection can be summarized as follows. Even if the explanation contains a law, this does not mean that the occurrence of the explanandum is the direct result of the occurrence of the fact mentioned in the explanans. It might also be the case, for example, that both facts are the result of the occurrence of a third, yet unknown, fact. In order for the explanation to genuinely count as a scientific explanation, it should therefore be made explicit that the latter fact is the cause of the former.

In pragma-dialectical terms, this additional requirement can be translated as the need to replace the arguments in the basic pattern that express the law(s) and the initial conditions that are part of the explanans $\left(1.1^{\prime} .1 \mathrm{a}\right.$ and $\left.1.1^{\prime} .1 \mathrm{~b}\right)$ by an argument of the form ' $Y$ causes $Z$ '. Since the critical questions associated with this type of argumentation relate to specific conditions under which a particular fact can be called the 'cause' of another fact, this argument may even be further supported by an argument addressing these criticisms. Such a subordinative argument may for instance express that without the occurrence of the cause, the effect does not occur either, in which case the supported argument expresses a necessary cause (if not Y, then not Z). Or it may express that there are no other factors needed for the effect to occur, in which case the supported argument expresses a sufficient cause (if $Y$, then $\mathrm{Z}$ ). In pragma-dialectical terms, this partial replacement and extension of the basic pattern can be represented as follows (Table 2).

Apart from institutional conventions regarding the quality of the explanation, the writings of philosophers of science also reflect conventions regarding the criteria that are used for selecting the best explanation from a number of candidate explanations. The type of reasoning involved in making this choice is known in the literature as 'abduction' or 'inference to the best explanation': 
Table 2 Extension concerning the quality of the explanation

\begin{tabular}{ll}
\hline $1.1^{\prime} .1$ & $\mathrm{Y}$ causes $\mathrm{Z}$ \\
$1.1^{\prime} .1 .1 \mathrm{a}$ & $\mathrm{Y}$ is a necessary cause of $\mathrm{Z}$ (if not $\mathrm{Y}$, then not $\mathrm{Z}$ ) \\
$1.1^{\prime} .1 .1 \mathrm{~b}$ & $\mathrm{Y}$ is a sufficient cause of $\mathrm{Z}$ (if $\mathrm{Y}$, then $\mathrm{Z}$ ) \\
\hline
\end{tabular}

In textbooks on epistemology or the philosophy of science, one often encounters something like the following as a formulation of abduction:

ABD1 Given evidence $E$ and candidate explanations $H_{1}, \ldots, H_{n}$ of $E$, infer the truth of that $H_{i}$ which best explains $E$.

An observation that is frequently made about this rule, and that points to a potential problem for it, is that it presupposes the notions of candidate explanation and best explanation, neither of which has a straightforward interpretation. While some still hope that the former can be spelled out in purely logical, or at least purely formal, terms, it is often said that the latter must appeal to the so-called theoretical virtues, like simplicity, generality, and coherence with well-established theories; the best explanation would then be the hypothesis which, on balance, does best with respect to these virtues. (Douven 2011, pp. 10-11)

From this and other accounts of abduction, in combination with a critical analysis of existing argumentation theoretical accounts of argument schemes that involve abduction and causal argumentation, I developed a "pattern of argumentation based on abduction' (Wagemans 2014). I will now use part of this pattern in order to describe the second type of extension of the basic pattern for justifying scientific explanations.

In many cases, the scientific explanation is presented as the result of a decisionmaking process in which several candidate explanations are taken into consideration. In anticipation to doubt or criticism regarding their choice of the best explanation from these candidate explanations, the basic pattern may be extended by arguments that function as a justification for this choice. This extension shares important characteristics with what is called 'pragmatic argumentation' in the pragma-dialectical literature, be it that in the scientific context, the main standpoint to be defended is descriptive rather than prescriptive in nature. In both cases, however, the sub-standpoint expressing the choice itself is of an evaluative nature. Within the scientific context, this sub-standpoint can be formulated as 'Of candidate explanations $H_{I}-H_{n}, H_{i}$ is the best explanation of $E^{\prime}$.

Given that making a choice always involves criteria on the basis of which it is decided which option is the best, the scientist may further support the substandpoint expressing the choice for the best explanation with arguments expressing the scores of the candidate explanations on the decision criteria employed. Such arguments may be represented in the form of a decision matrix of which the dimensions depend on the number of candidate explanations $\left(H_{I}-H_{n}\right)$ and the number of criteria $\left(C_{1}-C_{n}\right)$ and that contains the scores $\left(S_{1,1}-S_{n, n}\right)$ of the candidate explanations on these criteria (Table 3 ). 
Table 3 Decision matrix concerning the choice of the best explanation

\begin{tabular}{|c|c|c|c|c|}
\hline & $\boldsymbol{C}_{\boldsymbol{1}}$ & $\boldsymbol{C}_{2}$ & $\boldsymbol{C}_{3}$ & $\boldsymbol{C}_{\boldsymbol{4}}$ \\
\hline $\boldsymbol{H}_{\boldsymbol{1}}$ & $S_{1,1}$ & $S_{1,2}$ & $S_{1,3}$ & $S_{1,4}$ \\
\hline $\boldsymbol{H}_{2}$ & $S_{2,1}$ & $S_{2,2}$ & $S_{2,3}$ & $S_{2,4}$ \\
\hline $\boldsymbol{H}_{3}$ & $S_{3,1}$ & $S_{3,2}$ & $S_{3,3}$ & $S_{3,4}$ \\
\hline
\end{tabular}

The argument expressing the scores of the candidate explanations on the decision criteria employed can be formulated as follows: ' $H_{1}-H_{n}$ meet criteria $C_{1}-C_{n}$ with scores $S_{1,1}-S_{n, n}$ '.

As to the content of the criteria involved, Douven in the description of abduction as quoted above mentions three specific 'theoretical virtues' that may play a role in deciding what is the best explanation: 'simplicity, generality, and coherence with well-established theories'. From a survey of the literature on criteria for 'good' scientific theories it can be concluded that there are several other theoretical virtues and criteria and that their application in the decision-making process may vary considerably from one field to the other. The philosopher of science Kuhn, just to mention one other example, distinguishes between five criteria for evaluating the adequacy of a scientific theory:

Among a number of quite usual answers I select five, not because they are exhaustive, but because they are individually important and collectively sufficiently varied to indicate what is at stake ... These five characteristics - accuracy, consistency, scope, simplicity, and fruitfulness - are all standard criteria for evaluating the adequacy of a theory ... Together with others of much the same sort, they provide the shared basis for theory choice. (Kuhn 1998, p. 103, original italics)

Below I present the result of a survey of the literature (without claiming exhaustiveness of the list nor mutual exclusiveness of the categories). In this inventory, the criteria are subsumed under three different headings depending on their relation to three of the key aspects of the context in which the decision regarding what is the best scientific explanation takes place (Table 4).

A final extension of the basic pattern is based on the consideration that the scientist may support the choice of the best explanation by formulating or referring to a specific 'decision rule' on the basis of which it was decided how the scores on a selection of the abovementioned criteria have to be weighed or otherwise taken into account. The incorporation of such a decision rule in the extension of the pattern is prompted by a problem that is noted by, among others, Kuhn. The problem relates to the potential vagueness of the individual criteria and to the conflicts that may arise when there is more than one criterion involved in the decision:

Individually the criteria are imprecise: individuals may legitimately differ about their application to concrete cases. In addition, when deployed together, 
Table 4 Inventory of criteria for 'good' explanations

\begin{tabular}{ll}
\hline $\begin{array}{l}\text { Related to the observed } \\
\text { phenomenon }\end{array}$ & accuracy \\
& - scope, genericity, fruitfulness, explanatory force, subsumptive \\
& power \\
Related to the scientific context & - refutability, empirical content (testability, observability) \\
& - coherence \\
& - consistency \\
Related to competing explanations & - simplicity, elegance \\
& parsimony (fewest assumptions) \\
& - consilience (convergence of evidence) \\
\hline
\end{tabular}

they repeatedly prove to conflict with one another; accuracy may, for example, dictate the choice of one theory, scope the choice of its competitor. (Kuhn 1998, pp. 103-104)

Moreover, even if scientists agree on the criteria to be employed, they may attribute different weighing factors to them and therefore end up with a different decision: 'When scientists must choose between competing theories, two men fully committed to the same list of criteria for choice may nevertheless reach different conclusions' (Kuhn 1998, p. 105).

All in all, the second type of extension of the basic pattern for justifying scientific explanations can be identified as follows (Table 5).

Different from the situations to which the former extension applies, this extension applies to the situation in which there is more than one candidate explanation to choose from. As a consequence, the arguments that in the basic pattern function as arguments in support of the justificatory force of the main argument $\left(1.1^{\prime} .1 \mathrm{a}\right.$ and $\left.1.1^{\prime} .1 \mathrm{~b}\right)$ are already included in the decision matrix. For this reason, the sub-standpoint expressing the best explanation $\left(1.1^{\prime} .1\right)$ replaces instead of extends the part of the basic pattern containing the arguments expressing the law(s) (1.1'.1a) and initial conditions $\left(1.1^{\prime} .1 \mathrm{~b}\right)$.

Table 5 Extension concerning the choice of the best explanation

1.1'.1 Of candidate explanations $H_{1}-H_{n}, H_{i}$ is the best explanation of $E$

1.1'.1.1 $H_{1}-H_{n}$ meet criteria $C_{1}-C_{n}$ with scores $S_{I, I}-S_{n, n}$

1.1'.1.1' If $H_{1}-H_{n}$ meet criteria $C_{l}-C_{n}$ with scores $S_{l, l}-S_{n, n}$, then of possible explanations $H_{I}-H_{n}, H_{i}$ is the best explanation of $E$

1.1'.1.1'.1 Decision rule $R$ applies 


\section{Conclusion}

In this paper, I have identified an argumentative pattern for justifying scientific explanations. On the basis of accounts of the nature and shape of scientific explanations put forward by influential philosophers of science, I have described a basic argumentative pattern and two extensions of it. Taking the core elements of Hempel's two models of scientific explanation as a point of departure, I have proposed a basic pattern that has part of the explanans as the standpoint, the explanandum as the main argument, and the law(s) and initial conditions described in the models as arguments in support of the justificatory force of the main argument.

On the basis of specific criticisms regarding these models, I then proposed an extension of the pattern that applies to the situation in which the scientist provides arguments in support of the quality of the explanation. These arguments mainly reflect the requirement at force in several fields of expertise that the explanation should contain a cause in order to be genuinely called a scientific explanation.

Furthermore, on the basis of an account of the argumentative pattern reflecting the type of reasoning that is known as abduction, I proposed a second type of extension of the basic pattern. This extension relates to the institutional conventions regarding the criteria scientists employ in order to select the best explanation from a number of candidate explanations. Apart from the evaluative sub-standpoint expressing which explanation is the best, the extension consists of arguments expressing the criteria and the rule on which the choice is based. As to the criteria, I have presented the result of a survey of the literature concerning theoretical virtues and other types of criteria employed by scientists in determining whether a theory or an explanation is a good one. A future exploration of the theories for selecting and weighing these criteria as these are developed within decision theory may help identifying the specific decision rules involved.

From the identification of argumentative patterns in the scientific domain it may be concluded that pragmatic argumentation does not only play a role in justifying political, judicial or medical decisions, but also in justifying scientific explanations. Within the scientific domain, however, the main standpoint predominantly expresses a scientific explanation, which is descriptive in nature. As I have shown in this paper, pragmatic argumentation therefore only occurs on a lower level in the argumentative pattern. In particular, it may be expected in situations in which the scientist justifies the selection of the best scientific explanation from a number of candidate explanations.

Open Access This article is distributed under the terms of the Creative Commons Attribution 4.0 International License (http://creativecommons.org/licenses/by/4.0/), which permits unrestricted use, distribution, and reproduction in any medium, provided you give appropriate credit to the original author(s) and the source, provide a link to the Creative Commons license, and indicate if changes were made.

\section{References}

Curd, M., and J.A. Cover. 1998. Philosophy of science: The central issues. New York/London: Norton. Douven, I. 2011. Abduction. In The Stanford Encyclopedia of Philosophy (Spring 2011 Edition), ed. E.N. Zalta. http://plato.stanford.edu/archives/spr2011/entries/abduction/. 
Hacket, E.J., O. Amsterdamska, M. Lynch, and J. Wajcman (eds.). 2008. The handbook of science and technology studies, 3rd ed. Boston, MA/London: MIT Press.

Kuhn, T.S. 1998. Objectivity, value judgment, and theory choice. In Philosophy of science: The central issues, ed. Reprinted in M. Curd and J.A. Cover, 102-118. New York/London: Norton.

van Eemeren, F.H. Identifying argumentative patterns: A vital step in the development of pragmadialectics. Argumentation (to appear).

Wagemans, J.H.M. 2014. The assessment of argumentation based on abduction. In Virtues of argumentation: Proceedings of the 10th international conference of the Ontario Society for the Study of Argumentation (OSSA), 22-26 May 2013, ed. D. Mohammed, and M. Lewiński, 1-8. Windsor, ON: OSSA.

Wagemans, J.H.M. Criteria for deciding what is the 'best' scientific explanation. Proceedings of the ECA Lisbon 2015 Conference on Argumentation. (to appear). 